\title{
DETERMINING APPROPRIATE GOVERNMENT GUARANTEES FOR CONCESSION CONTRACT: LESSONS LEARNED FROM 10 PPP PROJECTS IN CHINA
}

\author{
Yelin XU ${ }^{a, *}$, John F. Y. YEUNG ${ }^{b}$, Shaohua JIANG ${ }^{c}$ \\ ${ }^{a}$ Department of Building and Real Estate, Zhejiang Sci-tech University, Hangzhou, China \\ ${ }^{b}$ Division of Business, College of International Education, School of Continuing Education, The Hong \\ Kong Baptist University, China \\ ${ }^{c}$ Department of Engineering Management, Dalian University of Technology, Dalian, China
}

Received 16 June 2013; accepted 29 September 2013

\begin{abstract}
Governments worldwide are keen to encourage PPP schemes, however PPP is not a foolproof business. Many local governments and private investors have suffered severe or trying experiences. In this research, 10 case studies were conducted to identify the obstacles and difficulties for successful implementation of PPP projects from the perspective of government guarantees. These PPP projects include two water supply plants, two sewage treatment plants, two power supply plants, two highway projects, and two bridge projects. The findings obtained indicate that the local governments are familiar with "the type of guarantees" to provide to the private investor but they are unsure of the "amount" that should be given. Many PPP projects have therefore been heavily criticized for over benefiting the private investors as the government provides them with too many guarantees. The inappropriate guarantees offered by the government are regarded as one of the root causes of PPP projects' failure. The lessons learned are valuable for determining appropriate government guarantees for future PPP projects and broadening the opportunities for infrastructure development with PPP procurement model.
\end{abstract}

KEYWORDS: Government guarantee; Public Private Partnership; Case study; Risk sharing

\section{INTRODUCTION}

Public Private Partnership (PPP) is a well-established procurement model for delivering public infrastructure projects in which "both the public and the private investor will contribute their expertise and resources to the project and share the risks involved" (Cheung et al. 2010). Successful implementation of PPP demands many prerequisites, such as a business-friendly environment, a wellestablished legal system, a clean administration and open markets (Sobhiyah et al. 2009). Given the high-risks involved and the lack of favorable environment needed, many local governments usually provide supports (government guarantees) in promoting the success of PPP projects to the extent allowed by laws, policies, and their capacities (Zhou 2004). However, determining a reasonable

\footnotetext{
* Corresponding author. E-mail: zjyelinxu@163.com
}

government guarantee for concession contract is a complicated issue. As too many guarantees provided by the government would decrease social welfare, increase project financing costs, and even induce government credit risks, while too few guarantees provided may make the project unfeasible in economic viability (Sobhiyah et al. 2009). Thus, a comprehensive and reasonable government guarantee structure is necessary for the success of PPP projects. This paper aims to take a closer look at the government guarantees in PPP projects through content analysis of cases, and explore management implications for determining appropriate government guarantees for concession contract. The study is believed to provide governments with practical experiences to design equitable risk sharing scheme and to benefit the private investor from effectively mitigating and managing risks in future PPP projects. 


\section{LITERATURE REVIEW}

The government's goal in launching PPP schemes is to facilitate infrastructure development under risks acceptable to the private investor who would in turn gain "reasonable but not excessive" returns (Zhang, Kumaraswamy 2001). However, privatization of infrastructure involves political, economic, legal, and environmental dimensions (Zhang 2005). To achieve a "win-win" result for both government and private investor, PPP requires favorable con- ditions. Longer-term uncertainties and wider-risk portfolios of PPP projects need substantial government guarantees to promote their success (Zhang, Kumaraswamy 2001). A comprehensive literature review (e.g. Lu 2006; Wang 2001; Liu 2004a) indicates that government guarantee may involve various aspects, such as political and legal guarantee, financing guarantee, construction and operation guarantee, and macro-economic environment guarantee as compiled in Table 1. Government guarantees have been extensively adopted in PPP projects

Table 1. A summary of government guarantees (GG) in PPP concession contract

\begin{tabular}{ll}
\hline $\begin{array}{l}\text { Political } \\
\text { and legal } \\
\text { guarantee }\end{array}$ & $\begin{array}{l}\text { GG1: Franchise right } \\
\text { guarantee }\end{array}$ \\
&
\end{tabular}

GG2: Legal risk guarantee

GG3: Administrative license guarantee

GG4: Free business guarantee

Financing guarantee

Construction and operation guarantee

Macro-economic environment guarantee

\section{GG5: Loan} guarantee

GG6: Provision of standby loans

GG7: Stock capital guarantee

GG8:

Non-competition guarantee

GG9: Raw material supply guarantee

GG10: Operating revenue guarantee

GG11: Force Majeure guarantee

GG12: logistical support guarantee

GG13: Tax preference guarantee

GG14: Inflation guarantee

GG15: Foreign exchange guarantee

GG16: Interest rate guarantee

Other

GG17: Comfort letter
The government promises don't retract franchise right in advance during the concession period. If, indeed required by public interests, the municipal government or its authorized department will provide reasonable reimbursement to the private investor.

The changes of legal environment will inevitably change the rights and obligations of PPP participants, and affect the economic anticipation of concessionaire. When the legal changes bring the private investor economic loss beyond a certain extent, the government promise to compensate the private investor's losses.

The local government promises that the project approval documents, such as government ratifications, licenses or consents needed in the development of projects, can be obtained smoothly.

The government permits the private investor to operate other facilities within the prescribed scope so as to ensure the private investor can gain some additional operating income.

The government provide guarantee to the bank for private investor's debts, and convince that the private investor has the capability to make repayments on time.

When the business income is reduced to a minimum due to some reasons, the government will provide standby loans to repay the prior debts for the private investor.

The host government promise to provide stock capital funds to the private investor.

The host government provides competition protection to the private investor so as to guarantee the anticipated income. For example, the government promises not to build a secondary competitive project in a certain times.

The government guarantees to provide the raw materials needed in project construction and/or operation to the private investor.

The government promises to provide the private investor with minimum income guarantees in the aspects of minimum purchase amount, minimum purchase price, or/and minimum investment earnings.

The government provides some financial support to offset the uninsurable force majeure risks for the private investor.

The host government promises to provide logistical support to the private investor, including land, labor forces, and relevant necessary infrastructures such as roads, electric wires and communication facilities needed in the construction and operation of PPP projects.

The local government promise to provide the private investor with guaranties on tax reduction and exemption.

When inflation exceeds the agreed margin, the government guarantees to reimburse the private investor by adjusting the concession price or/and extending the concession period.

The government promises that the investors can exchange the project income into convertible currency and remit it abroad. If the exchange rate fluctuation exceeds the agreed margin, the government guarantees to share the losses incurred from the fluctuations of exchange rate, and provide investors with economic reimbursement.

The host government provides the private investor with interest rate guarantee. When the increase of interest rate exceeds the prescribed percentage within the concession period, the private investor will obtain reimbursement from the government.

The comfort letter issued by the government is not legally binding, but is important in project financing as an intention guarantee.

\footnotetext{
* Adopted from Lu (2006), Wang (2001) and Liu (2004b).
} 
across countries and sectors, such as North-South highway project in Malaysia, Karachi power plant in Pakistan (Kumaraswamy, Zhang 2001), Laibin B power plant in China (Wang, Tiong 2000), Rudeshur gas turbine power station in Iran (Sobhiyah et al. 2009), Huaibei power plant in China (Smith et al. 2004), and harbor tunnel in Sydney (Yeo 2003).

Early project practices also indicate that government guarantee is not only an important factor for improving the enthusiasm of private capital to participate in PPP projects, but can also greatly affect a PPP project's financial feasibility (Deng 2007). Take one of the most classic cases in the world, Channel Tunnel as an example, in order to enhance the project's resistibility to market risks, the UK and French government provided a series of guarantees to the private investor to ensure the tunnel's financial viability (Ke et al. 2008). These guarantees include: 1) competition protection guarantee: no-secondary facility will be built in next 33 years; 2) commercial independence guarantee: grant concession pricing right to the private investor; 3) long-term sales guarantee: establish interconnection with the railway network. Ke et al. (2008) considered that government guarantees adopted in this project set a good example for the implementation of subsequent PPP projects in the past decades. Despite the construction cost was overspent due to geological conditions and the present operating status is still not optimistic, the method for dealing with huge risks through government guarantee is appraisable.

Reasonable government guarantee structure is vital to PPP project's success. However, if the government guarantee is inequitably or inappropriately provided, or beyond the capacity of the government, PPP projects would fail (Xu 2010). A failing PPP project may further deter investors from investing in similar projects or in the same area and ultimately, the host government will suffer a greater loss in economic and social development (Tam 1999). Moreover, as the application of PPP in many areas hasn't been fully developed yet, many local governments lack necessary knowledge in PPP procurement and have less experience of concession projects than private investors (Deng 2007). The insufficiency of information can easily induce the governments to make undue or wrong decisions on government guarantees (Deng 2007). Take the most famous project, Sha Jiao B power plant in China as an example, the government provided excessive guarantees to Hehe power Ltd. of Hong Kong for attracting private capital, which caused the government to assume 16.8 billion HK Dollar of exchange rate losses and 4.7 billion CNY of fuel cost escalation losses, while the private investor's internal rate of return during the concession period reached 38.8\% (Cao 2006).

In order to take a closer look at the government guarantees in PPP projects, the remainder of the paper is organized as follows. The third section presents the background of 10 cases and the following fourth section deals with the relationship between government guarantees and corresponding project performance. Afterwards, the analysis and discussion of the ten case studies is presented. Finally the last section provides the conclusion remarks.

\section{BACKGROUND OF SELECTED CASES}

Case studies were selected as an appropriate mean for the research in identifying how government guarantees influence the success of PPP projects. It is considered the preferred research strategy when a "how" or "why" question is being asked about a contemporary set of events over which the investigator has little or no control (Laishram, Satyanarayana 2009; Yin 2009). A total of 34 PPP projects were first collected from journal papers, doctoral/master thesis, and news reports from official media. 10 of them were finally selected and studied in this study as they contained all the information as required according to the project selection criteria as follows:

(1) The projects have detailed information on government guarantees and their corresponding project performance can serve the purpose for an in-depth investigation;

(2) The projects cover different types;

(3) The projects are distributed in different cities and operated under different social, economical, political and legal environments;

(4) The data obtained can be triangulated to ensure the internal validity of the results (Yin 2009).

Their background information is summarized in Table 2. To improve the generality of research findings, these cases were selected from five different project types including water supply, sewage treatment, power supply, highway, and bridge as PPPs are widely used in these sectors.

\section{GOVERNMENT GUARANTEES AND CORRESPONDING PROJECT PERFORMANCE OF SELECTED CASES}

This section illustrates government guarantees provided in the above ten cases and their correspond- 
Table 2. Background information of PPP cases selected

\begin{tabular}{|c|c|c|c|c|c|c|c|c|c|}
\hline $\begin{array}{l}\text { Case } \\
\text { No. }\end{array}$ & $\begin{array}{l}\text { Project name/ } \\
\text { location }\end{array}$ & $\begin{array}{l}\text { Project } \\
\text { type }\end{array}$ & Project scale & $\begin{array}{l}\text { Total } \\
\text { investment }\end{array}$ & $\begin{array}{l}\text { Construction } \\
\text { period }\end{array}$ & $\begin{array}{l}\text { Concession } \\
\text { period }\end{array}$ & $\begin{array}{l}\text { Concession- } \\
\text { aire }\end{array}$ & $\begin{array}{l}\text { Public } \\
\text { sector }\end{array}$ & Source \\
\hline 1 & $\begin{array}{l}\text { No. } 6 \text { water } \\
\text { plant of Cheng- } \\
\text { du, China }\end{array}$ & $\begin{array}{l}\text { Water } \\
\text { supply }\end{array}$ & $\begin{array}{l}400 \text { thou- } \\
\text { sand } \mathrm{m} 3 / \text { day }\end{array}$ & $\begin{array}{l}106.5 \text { mil- } \\
\text { lion USD }\end{array}$ & 2.5 years & 18 years & $\begin{array}{l}\text { Chengdu } \\
\text { Tongyong } \\
\text { water group }\end{array}$ & $\begin{array}{l}\text { Chengdu } \\
\text { municipal } \\
\text { government }\end{array}$ & $\mathrm{Xu}(2004)$ \\
\hline 2 & $\begin{array}{l}\text { No. } 9 \text { water } \\
\text { plant of Shen- } \\
\text { yang, China }\end{array}$ & $\begin{array}{l}\text { Water } \\
\text { supply }\end{array}$ & $\begin{array}{l}100 \text { thou- } \\
\text { sand m3/day }\end{array}$ & $\begin{array}{l}25 \text { million } \\
\text { USD }\end{array}$ & 1 year & 20 years & $\begin{array}{l}\text { Hong Kong } \\
\text { Huijin Ltd. }\end{array}$ & $\begin{array}{l}\text { Shenyang } \\
\text { water com- } \\
\text { pany }\end{array}$ & $\begin{array}{l}\text { Liu } \\
(2004)\end{array}$ \\
\hline 3 & $\begin{array}{l}\text { Bei Jiao sew- } \\
\text { age treatment } \\
\text { plant, China }\end{array}$ & $\begin{array}{l}\text { Sewage } \\
\text { treatment }\end{array}$ & $\begin{array}{l}50 \text { thousand } \\
\mathrm{km} 3 / \text { day }\end{array}$ & $\begin{array}{l}10 \text { million } \\
\text { USD }\end{array}$ & 1.5 years & 20 years & $\begin{array}{l}\text { Beijiao sew- } \\
\text { age treat- } \\
\text { ment Ltd. }\end{array}$ & $\begin{array}{l}\text { Local mu- } \\
\text { nicipal } \\
\text { government }\end{array}$ & $\mathrm{Lu}(2006)$ \\
\hline 4 & $\begin{array}{l}\text { HuiJin sew- } \\
\text { age plant of } \\
\text { ChangCun, } \\
\text { China }\end{array}$ & $\begin{array}{l}\text { Sewage } \\
\text { treatment }\end{array}$ & $\begin{array}{l}390 \text { thou- } \\
\text { sand } \mathrm{km} 3 / \\
\text { day }\end{array}$ & $\begin{array}{l}39.7 \text { million } \\
\text { USD }\end{array}$ & 1 year & 21 years & Huijing Ltd. & $\begin{array}{l}\text { Changcun } \\
\text { municipal } \\
\text { government }\end{array}$ & $\mathrm{Zi} \mathrm{(2005)}$ \\
\hline 5 & $\begin{array}{l}\text { Laibin B power } \\
\text { plant, China }\end{array}$ & $\begin{array}{l}\text { Power } \\
\text { supply }\end{array}$ & $\begin{array}{l}360 \text { thou- } \\
\text { sand } \\
K W H \times 2\end{array}$ & $\begin{array}{l}620 \text { million } \\
\text { USD }\end{array}$ & $\begin{array}{l}2 \text { years } \\
\text { and } 9 \\
\text { months }\end{array}$ & 18 years & $\begin{array}{l}\text { EDF consor- } \\
\text { tium }\end{array}$ & $\begin{array}{l}\text { Guangxi } \\
\text { Zhuang } \\
\text { autonomous } \\
\text { government }\end{array}$ & $\begin{array}{l}\text { Zhou } \\
(2004)\end{array}$ \\
\hline 6 & $\begin{array}{l}\text { Sha Jiao B } \\
\text { power plant, } \\
\text { China }\end{array}$ & $\begin{array}{l}\text { Power } \\
\text { supply }\end{array}$ & $\begin{array}{l}350 \text { thou- } \\
\text { sand } \\
K W H \times 2\end{array}$ & $\begin{array}{l}540 \text { million } \\
\text { USD }\end{array}$ & 3 years & 13 years & $\begin{array}{l}\text { Hehe power } \\
\text { Ltd. of } \\
\text { HongKong }\end{array}$ & $\begin{array}{l}\text { Guangshen } \\
\text { Shajiao } \\
\text { power Ltd. }\end{array}$ & $\begin{array}{l}\text { Cao } \\
(2006)\end{array}$ \\
\hline 7 & $\begin{array}{l}\text { Xiang Fan } \\
\text { Highway of } \mathrm{Hu} \\
\text { Bei, China }\end{array}$ & Highway & $185.4 \mathrm{~km}$ & $\begin{array}{l}541 \text { million } \\
\text { USD }\end{array}$ & 3.5 years & 35 years & $\begin{array}{l}\text { Xiangfan } \\
\text { highway } \\
\text { Ltd. }\end{array}$ & $\begin{array}{l}\text { Xiang Fan } \\
\text { municipal } \\
\text { government }\end{array}$ & $\begin{array}{l}\text { Zhao } \\
(2002)\end{array}$ \\
\hline 8 & $\begin{array}{l}\text { An-Jing High- } \\
\text { way of Yun- } \\
\text { Nan, China }\end{array}$ & Highway & $39 \mathrm{~km}$ & $\begin{array}{l}210 \text { million } \\
\text { USD }\end{array}$ & 2 years & 26 years & $\begin{array}{l}\text { China rail- } \\
\text { way } \\
\text { construc- } \\
\text { tion } \\
\text { corporation } \\
\text { Ltd. }\end{array}$ & $\begin{array}{l}\text { Kunming } \\
\text { municipal } \\
\text { government }\end{array}$ & $\begin{array}{l}\mathrm{Ma} \\
(2004)\end{array}$ \\
\hline 9 & $\begin{array}{l}\text { CiTong bridge } \\
\text { of Quan Zhou, } \\
\text { China }\end{array}$ & Bridge & $1.530 \mathrm{~km}$ & $\begin{array}{l}36 \text { million } \\
\text { USD }\end{array}$ & 1.5 years & 30 years & $\begin{array}{l}\text { CiTong } \\
\text { bridge Ltd }\end{array}$ & $\begin{array}{l}\text { Quanzhou } \\
\text { municipal } \\
\text { government }\end{array}$ & Ji (2006) \\
\hline 10 & $\begin{array}{l}\text { Nanjing No. } 3 \\
\text { Yangtze River } \\
\text { Bridge, China }\end{array}$ & Bridge & $4.744 \mathrm{~km}$ & $\begin{array}{l}454 \text { million } \\
\text { USD }\end{array}$ & 2.5 years & 32.5 years & $\begin{array}{l}\text { Nanjing } \\
\text { No. } 3 \text { Yang- } \\
\text { tze River } \\
\text { Bridge Ltd. }\end{array}$ & $\begin{array}{l}\text { Nanjing } \\
\text { municipal } \\
\text { government }\end{array}$ & $\mathrm{Li}(2006)$ \\
\hline
\end{tabular}

ing project performances to present the impact of government guarantee on PPP projects' implementation. Various types of information on the 10 projects have been collected. Data collected in different means were thoroughly analyzed by content analysis, which contains a systematic analytic process of three steps, (1) identification of government guarantees provided in each project, (2) identification of their corresponding project performances, and (3) analysis of the relationship between government guarantees and their corresponding project performance (Song et al. 2013). The coding system for government guarantees, as shown in Table 3, follows the coding order in Table 1.

Seven out of the ten cases appear to be failure or less successful (as local government assumed excessive responsibilities, which actually exceed their risk management and sustainment capability). Three are seen as successful, or at least, as most of the project stakeholders are happy with their project performances. The criteria for measuring project success include three dimensions (Khang, Moe 2008): (1) The efficiency of the implementation process (time, cost, technical goals and working relationship); (2) Products and service quality of PPP projects; (3) The satisfaction of stakeholders. To simplify the information of above projects, government guarantees and their corresponding PPP project performances were extracted and summarized in Table 3.

\section{LESSONS LEARNED FROM CASES}

To sort the findings reported above, the government guarantee can be classified into three categories.

(1) The government provided excessive guarantees to the private investor.

(2) The government provided appropriate guarantees to the private investor.

(3) The government provided insufficient guarantees to the private investor. 


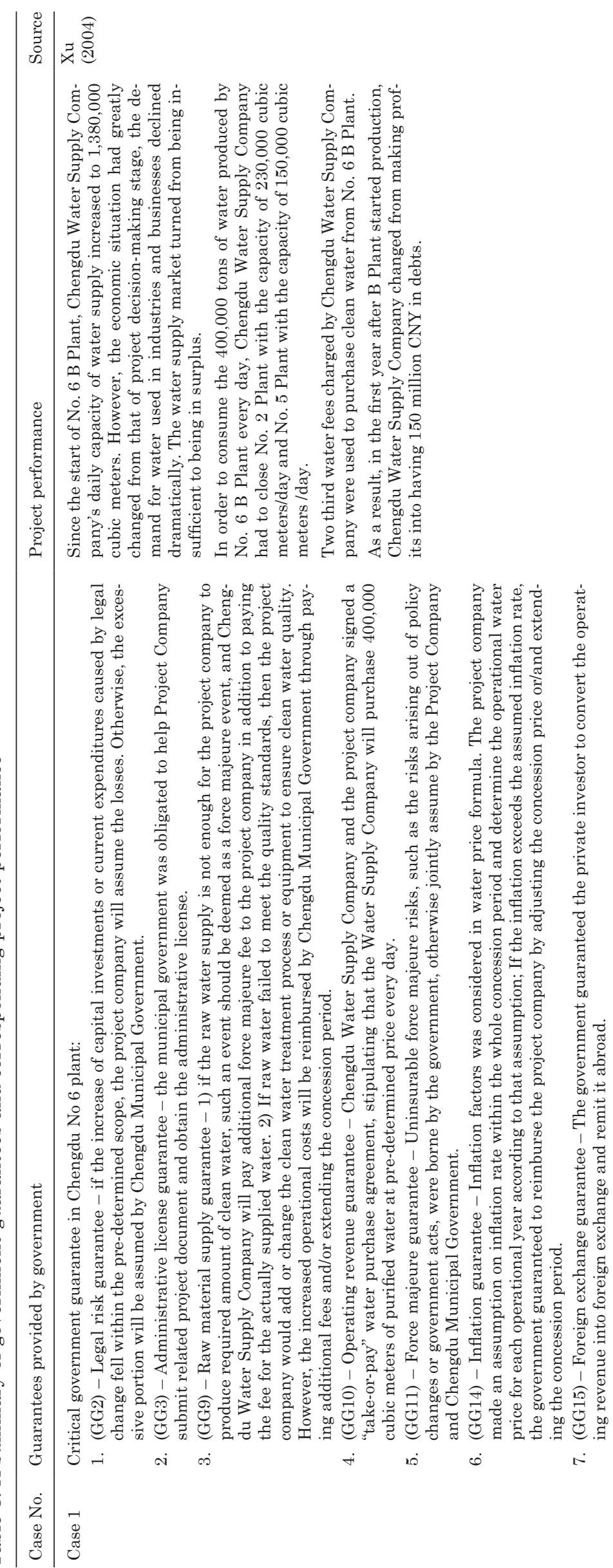

: :

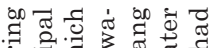

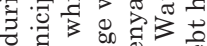

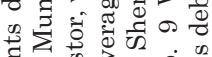

政

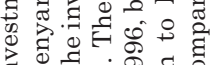

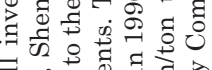

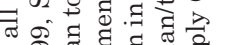

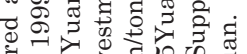

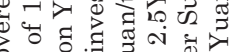

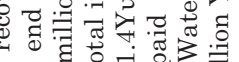

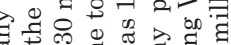

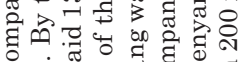

ช

3 政西

up

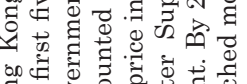

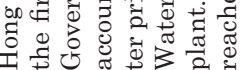

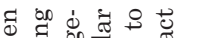

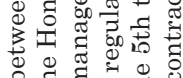

过 월

菏

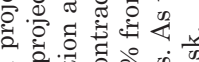

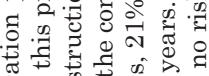
कृ.

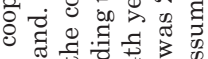
里

an

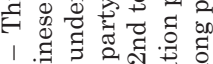

든

在

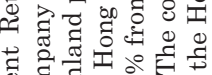

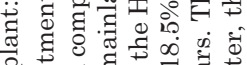

20

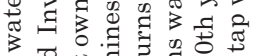

5.

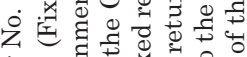

of o s

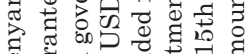

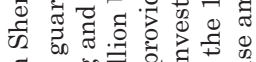

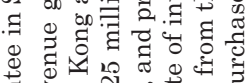

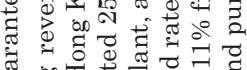

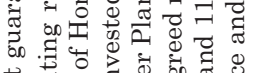

可

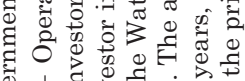
1 . on 0 \%

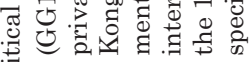

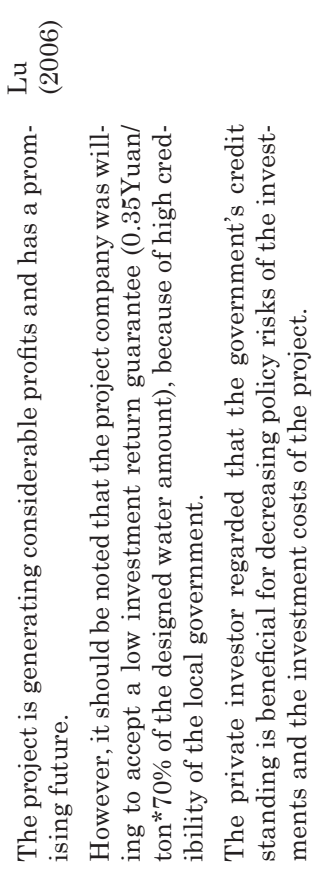

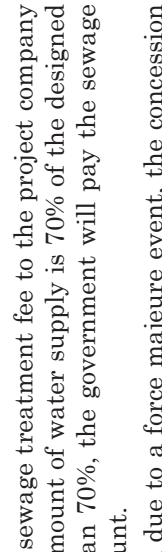

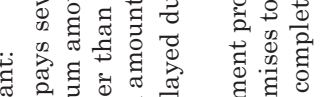

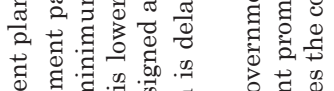
च च :

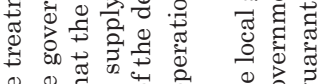

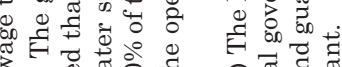

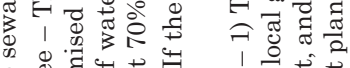

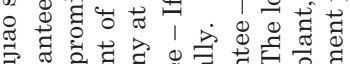

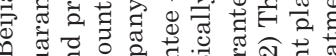

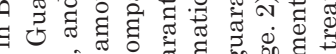
ه

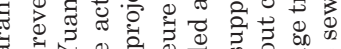
菏

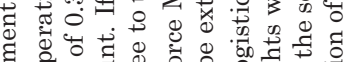

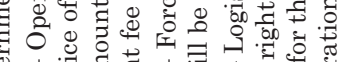

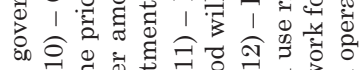

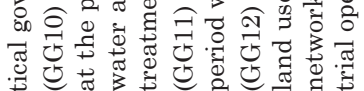
प्रु $\rightarrow$ का

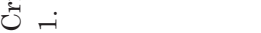




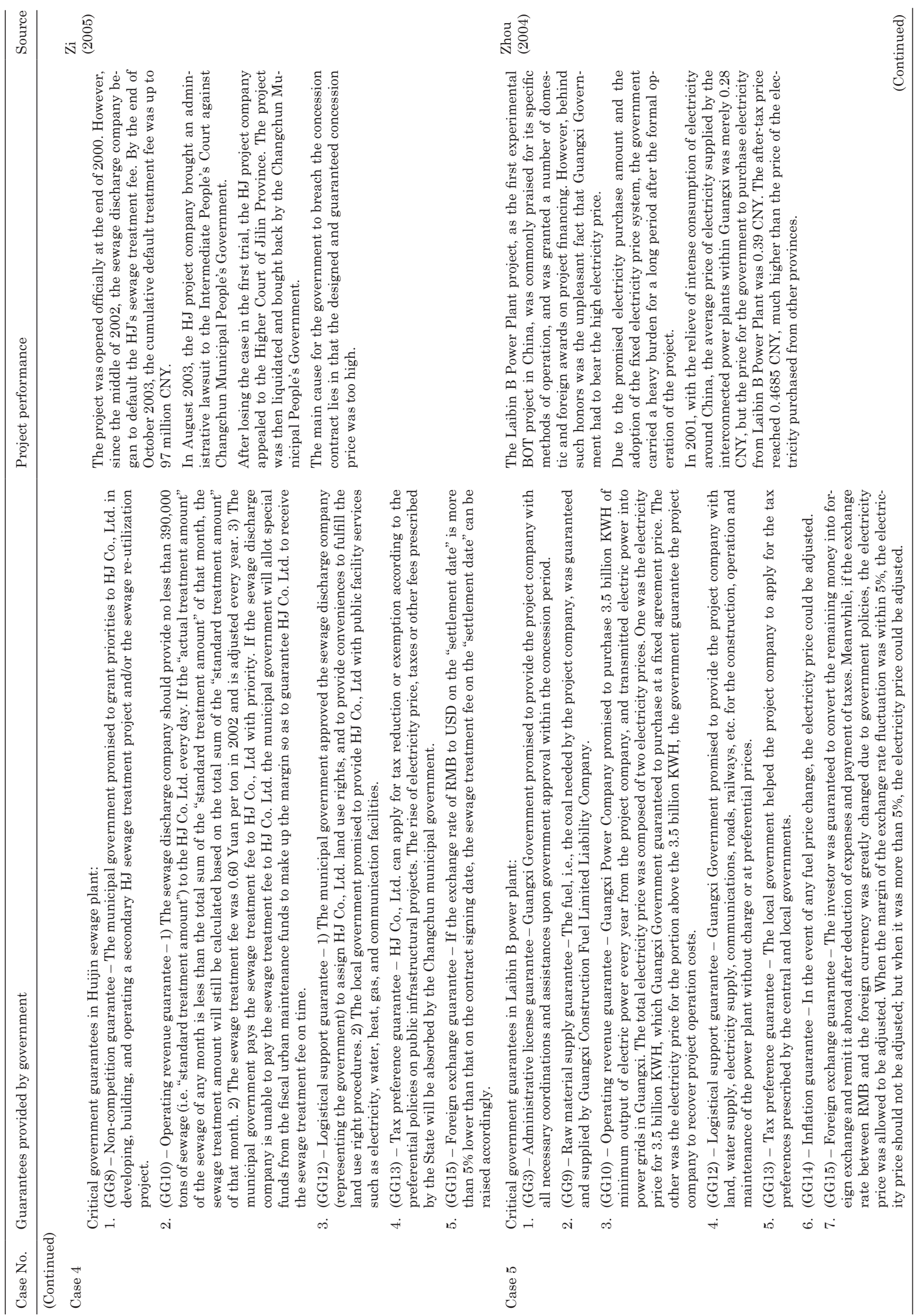



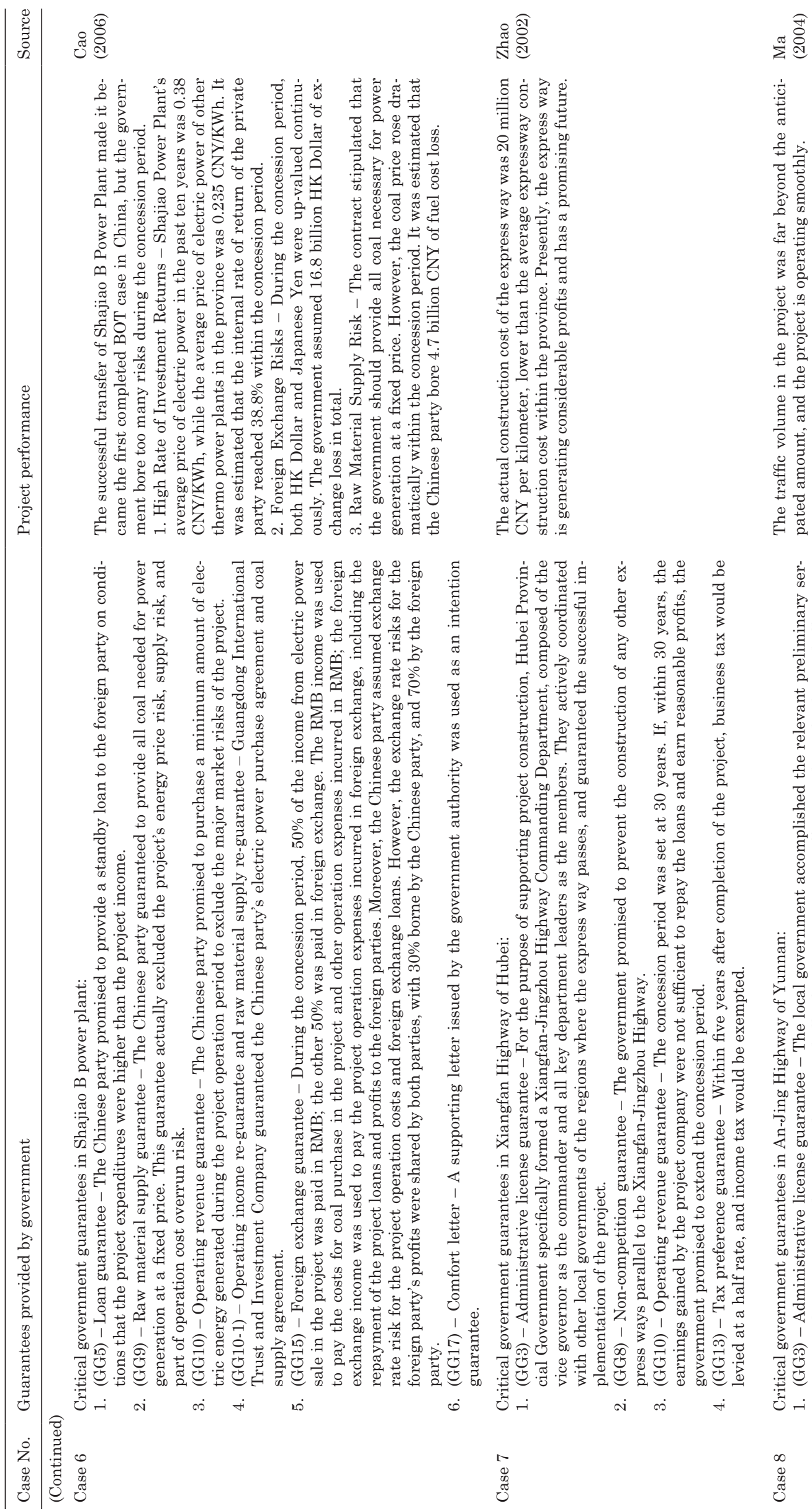

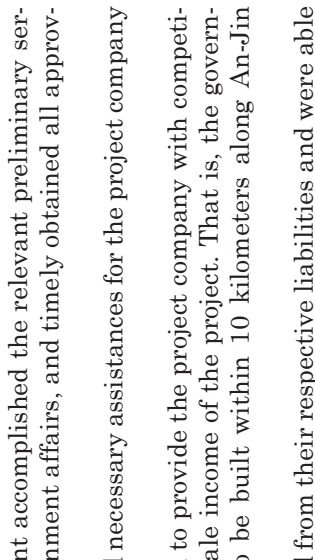

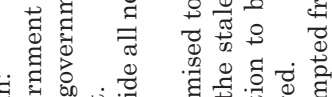

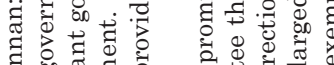

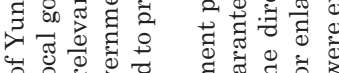

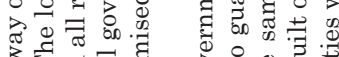

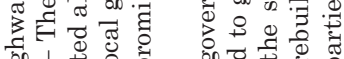

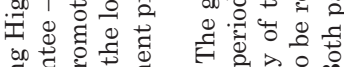

on

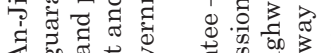

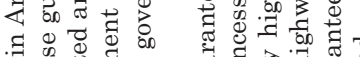

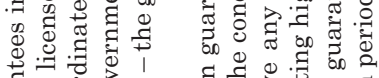

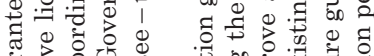

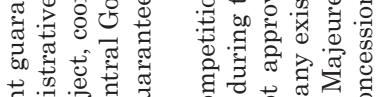

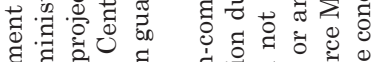

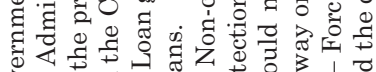

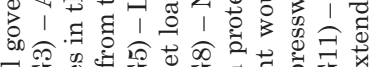

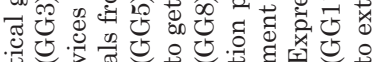

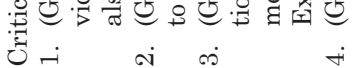




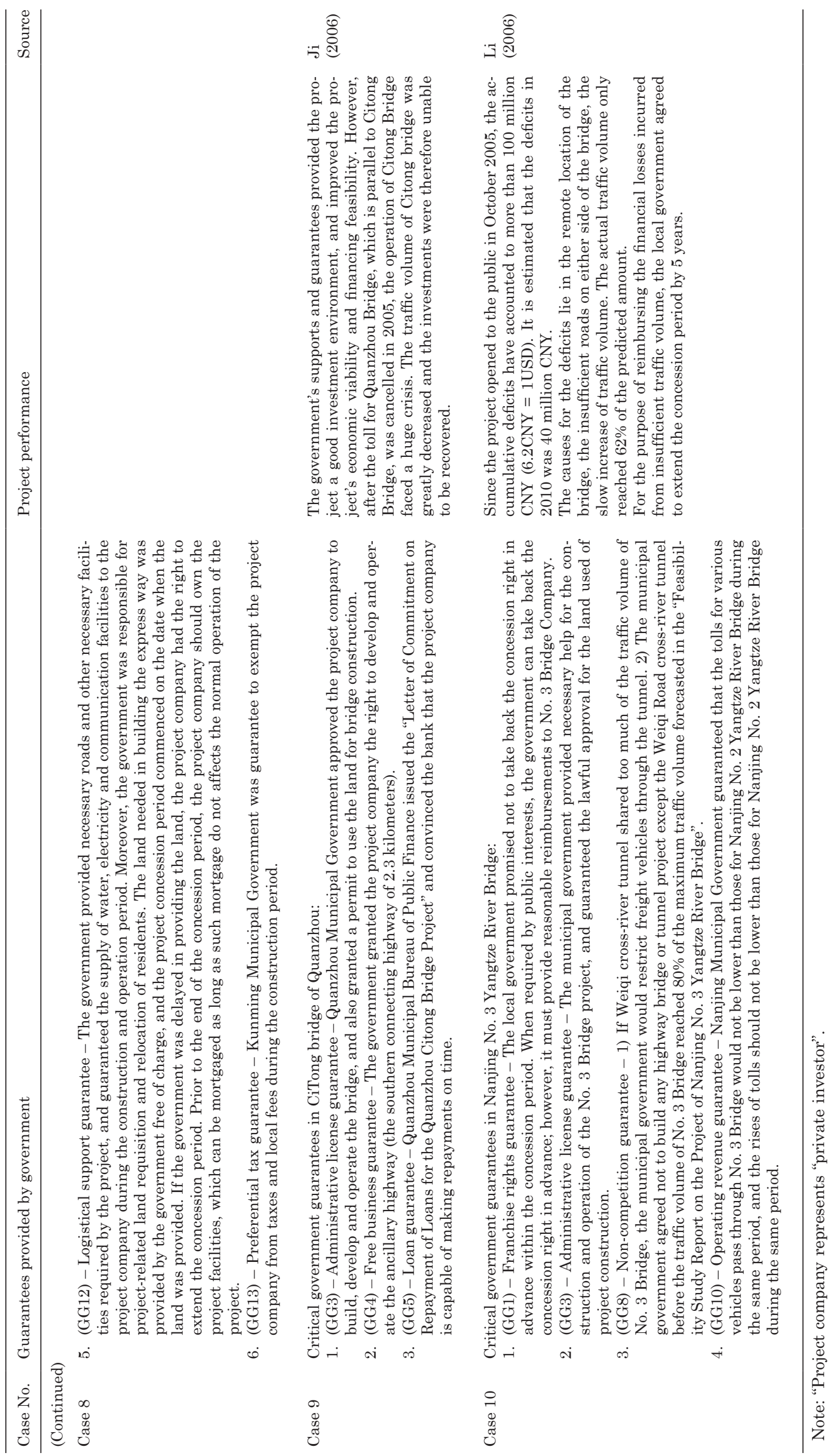


An analysis of project performance indicates that the government guarantees provided in six cases out of the ten were inappropriate (excessive or insufficient). Five out of six cases provided excessive guarantees to the private investor and one provide inadequate support to its concessionaire as compiled in Table 4.

Table 4 indicates that governments assumed excessive responsibilities in half of PPP projects, which actually exceed their risk management and sustainment capability. It may be due to the fact that developing market/government, with imperfect legal and regulation systems and unstable market environments and greater investment risks, tends to make excessive commitments and guarantees to the private party for the purpose of attracting private funding (Deng 2007). However, such unreasonable government guarantees led to unsuccessful project performance to the PPP scheme as shown in the following cases:

- In Case 1, Chengdu Municipal Government not only assumed political and legal change risks but also bore market risk, raw water supply risk, and partial exchange rate risk. The risk sharing mechanism in this project is significantly unreasonable. Generally, a rea- sonable sharing structure is that the private investor bears the price risk of raw water, while the market risks, directly arising from price fluctuations, were shared between the government and the private investor through a price adjustment mechanism. The private investor could transfer or mitigate such risks through signing a long-term energy and raw material supply agreement with the supplier. However, in this project, raw water supply risk was solely assumed by Chengdu Municipal Government, as well as all operating revenue risks. This resulted in the Chengdu Water Supply Company to use two third of the water fee revenue to pay for B Plant's clean water (Xu 2004).

- In Case 2, the average water price in Shenyang was 1.4 Yuan/ton in 1996, but Shenyang Water Supply Company paid 2.5 Yuan/ton to No. 9 Water plant. Since the government provided excessive guarantee on fixed rate of investment returns, the private investor recovered all principal of investments during the first five years, and would continue to recover profits in following 15 years (Liu 2004b).

- In Case 4, the government provided the private investor a purchase guarantee of no less

Table 4. Appropriateness of government guarantees provided in ten cases

\begin{tabular}{|c|c|c|c|c|c|}
\hline \multirow{2}{*}{$\begin{array}{l}\text { Case } \\
\text { No. }\end{array}$} & \multirow[t]{2}{*}{ Project name/location } & \multicolumn{3}{|c|}{ Government guarantee } & \multirow[t]{2}{*}{ Note } \\
\hline & & Excessive & Appropriate & Insufficient & \\
\hline 1 & $\begin{array}{l}\text { No. } 6 \text { water plant of Chengdu, } \\
\text { China }\end{array}$ & $\times$ & & & $\begin{array}{l}\text { Excessive operating income } \\
\text { guarantee Excessive raw material } \\
\text { supply guarantee }\end{array}$ \\
\hline 2 & $\begin{array}{l}\text { No. } 9 \text { water plant of Shenyang, } \\
\text { China }\end{array}$ & $\times$ & & & $\begin{array}{l}\text { Excessive operating income } \\
\text { guarantee }\end{array}$ \\
\hline 3 & $\begin{array}{l}\text { Bei Jiao sewage treatment } \\
\text { plant, China }\end{array}$ & & $x$ & & \\
\hline 4 & $\begin{array}{l}\text { HuiJin sewage plant of Chang- } \\
\text { Cun, China }\end{array}$ & $\times$ & & & $\begin{array}{l}\text { Excessive operating income } \\
\text { guarantee }\end{array}$ \\
\hline 5 & Laibin B power plant, China & $\times$ & & & $\begin{array}{l}\text { Excessive operating income } \\
\text { guarantee }\end{array}$ \\
\hline 6 & Sha Jiao B power plant, China & $\times$ & & & $\begin{array}{l}\text { Excessive operating income } \\
\text { guarantee } \\
\text { Excessive foreign exchange } \\
\text { guarantee } \\
\text { Excessive raw material supply } \\
\text { guarantee }\end{array}$ \\
\hline 7 & $\begin{array}{l}\text { Xiang Fan Highway of Hu Bei, } \\
\text { China }\end{array}$ & & $\times$ & & \\
\hline 8 & $\begin{array}{l}\text { An-Jing Highway of YunNan, } \\
\text { China }\end{array}$ & & $\times$ & & \\
\hline 9 & $\begin{array}{l}\text { CiTong bridge of Quan Zhou, } \\
\text { China }\end{array}$ & & & $x$ & Inadequate competition protection \\
\hline 10 & $\begin{array}{l}\text { Nanjing No. } 3 \text { Yangtze River } \\
\text { Bridge, China }\end{array}$ & & $\times$ & & \\
\hline
\end{tabular}


than 390,000 tons of sewage at the price of 0.60 Yuan per ton. The high revenue guarantee results in the sewage discharge company, representing the government, was unable to pay the sewage treatment fee to the private investor within two years of operation ( $\mathrm{Zi}$ 2005).

- In Case 5, Guangxi Government promised Laibin B Power Plant to purchase 3.5 billion KWH of electricity per year at the price of 0.39 Yuan per KWH. However, the average price of electricity supplied by the other power plants in Guangxi province was just 0.28 Yuan. The excessive price guarantee became a millstone round the neck of the local government (Zhou 2004).

- In Case 6, the private investor's internal rate of return during the concession period reached $38.8 \%$. While the government assumed 16.8 billion HK Dollar of exchange rate losses and 4.7 billion $\mathrm{CNY}$ of fuel cost escalation losses (Cao 2006).

Government guarantees can reduce risk but are not free of cost (Wibowo et al. 2012; Wibowo 2004). Excessive guarantees will lead to high performance costs for the government and prevent the concession agreement to be fulfilled smoothly. This has been verified by the above cases.

The governments usually do not know the full extent of contingent liabilities when providing guarantees, because they account and record guarantee costs only when guarantees come due (Wibowo 2004). In above five cases, operating revenue guarantee is the first risky government guarantee, which needs both sides' special attention, as nearly all projects with excessive revenue guarantees eventually fail or do not succeed. The second risky guarantee is raw material supply and the third is foreign exchange guarantee as shown in Table 4. According to the principle of risk allocation, risks should be allocated to the party best able to handle them, i.e. the risk bearer should be the one most able to prevent risk from occurring and able to minimize the consequences if risk does occur. However, it has never been easy to obtain an equitable risk sharing, as so many other factors also have strong impacts on it, such as bargaining power and negotiation tactics of practitioners, risk attitude, market compulsion caused by competition, cooperation history, and so on (Lam 1999). At the present, many local governments in China starve for private capital and lack necessary expertise and experience in PPP implementation. They usually tend to offer excessive preferential conditions to the private investors for attracting private funding by promising fixed rate of investment returns, minimum purchase amount, and fixed price of products or services. These guarantees not only lead the private investor to have a high expectation on investment return rate, but also make the private investor less motivated to be efficient in project management or/and risk management because it believes that the local government will provide a downside protection for risk losses (Wibowo 2004). However, the fulfillment of such unreasonable government guarantee would be greatly influenced by the change of session of the local government, the conflict between central policies and local ones, and the change of market environment (Deng 2007). Thus, the rate for government's breach of contract is rather high and many projects were therefore liquidated, such as that in case 4 .

Compared to developing markets, developed markets or regions have relatively mature markets, sound legal systems, and fewer state risks. In the developed markets, governments are usually only willing to bear the systematic risks and necessary commercial risks relevant to the projects (Zhao 2002). Take Hong Kong tunnel as an example, the franchise consortia of HK tunnel assumed risks related to construction, engineering, geology, environment, climate, financing, inflation, and cost escalation. The government provided guarantee on the risk of land acquisition; however, no guarantees or warranties are given with regard to the program for commencement or completion, tax exemption, minimum traffic flows, economic returns, and future competitive routes (Zhang, Kumaraswamy 2001). Similar projects located in western countries also have similar government guarantee structure.

The practices of above five cases confirmed that excessive guarantee would result in difficulties for the government to perform its obligation, and may even lead to project failure. However, inadequate government guarantee may also have a similar negative impact on project's implementation.

- Case 9: the government cancelled the toll for Quanzhou Bridge, which is parallel to Citong Bridge from 2005. The operating revenue of Citong Bridge is then declined immediately, and the private investor faced a huge financial crisis. It was evaluated that Citong Bridge's original investment recovery goal was unable to be achieved (Ji 2006).

Different from the above six failed cases, the government guarantee provided in Case 3 are relatively reasonable. Due to the good reputation of local government (means lower credit risk), the 
private investor requested a relatively reasonable investment return guarantee i.e. $70 \%$ of the designed water amount at a price of 0.35 Yuan/ton. The relatively equitable risk sharing promoted the success of this project to some extent. The project is currently operating smoothly. This case implies that the local government's high credibility may be beneficial for mitigating the investment risks and decreasing the private investor's requisition on government guarantee ( $\mathrm{Lu} 2006)$. Wibowo (2004) and Irwin et al. (1999) also considered that one of the bests that a government can do to make a project more attractive without providing excessive guarantees is to formulate good policies and establish a favorable environment that generally reduce risks and raise expected returns.

In addition, the performances of cases 7,8 , and 10 indicate that the risk of the project itself is still the key factor for the government to fulfill the contract. No matter how enthusiastic the government is and how attractive its guarantees are, sufficient market study is still the perquisite to the success of PPP projects (Wang, Tiong 2000). For example, due to inaccurate market forecast, Nanjing No. 3 Yangtze River Bridge of case 10 suffered a huge market risks. Although the government provided concession price guarantee and non-competition protection, the actual traffic volume only reached $62 \%$ of the designed volume due to the remote location of the bridge, insufficient roads that connect the bridge, and the slow increase of traffic volume (Li 2006). Since it was open to the public in October 2005, the cumulative deficits of the project have reached more than 15 million USD in past four years ( $\mathrm{Li}$ 2006). On the contrary, the actual traffic volume of cases 7 and 8 are far more than anticipated; the projects are receiving considerable profits and have promising futures (Zhao 2002; Ma 2004). These three cases imply that government guarantees should not be deemed the only tool to ensure the success of PPP projects, which is more greatly dependent on the financial viability of projects themselves.

\section{CONCLUSIONS}

PPP model offers a viable vehicle for accelerating the pace of infrastructure construction and economic development. However, not all projects are successful. In this study, ten typical PPP examples were selected and analyzed to explore root causes behind successful implementation of PPP projects from the perspective of government guarantee.
Three valuable experiences were learnt, which include:

1. There are a total of 17 different government guarantees available. However, operating revenue guarantee, raw material supply guarantee, foreign exchange guarantee and competition protection guarantee are the four most common guarantees adopted in China's PPP concession contracts.

2. An analysis of 10 PPP cases reinforces that government guarantees can reduce risk but are not free of cost. Excessive guarantees will lead to high performance costs for the government and prevent the concession agreement to be fulfilled smoothly.

3. The economic viability of the project self, instead of government guarantees is the key factor for the success of PPP projects. Well-established regulatory framework and favorable project implementation environment are beneficial to decrease the private investor's requirement on government guarantees.

The result from the case studies indicate that both private and public sectors need to have a better understanding of risk sharing in order to develop a reasonable guarantee mechanism/structure and enable the project to generate better outcomes. Moreover, there is a limitation in this research. The case studied may not precisely reflect the generalized features of PPP projects in China. Further research is needed to verify the research results obtained.

\section{ACKNOWLEDGEMENTS}

The work described in this paper was supported by the National Natural Science Foundation of China (71471166 and 71101130), Qianjiang Talent Project of Zhejiang (QJC1302019) and Science Foundation of the Zhejiang Sci-Tech University (1205827-Y and 521 talent project).

\section{REFERENCES}

Cheung, E.; Chan, A.; Kajewski, S. 2010. The public sector's perspective on procuring public works projects comparing the views of practitioners in Hong Kong and Australia, Journal of Civil Engineering and Management 16(1): 19-32. http://dx.doi.org/10.3846/ jcem.2010.02

Cao, T. 2006. BOT financing research - take ShaJiao B power plant as an example: Excellent Master Thesis. Jinan University. (In Chinese) 
Deng, X. P. 2007. Research on risk allocation and countermeasures in implementing PPP projects: $\mathrm{PhD}$ Thesis. Southeast University. (In Chinese)

Irwin, T.; Klein, M.; Perry, G.; Thobani, M. 1999. Managing government exposure to private infrastructure risks, The World Bank Research Observer 14(2): 229245. http://dx.doi.org/10.1093/wbro/14.2.229

Ji, F. 2006. Study on mode and risk of BOT project $f-$ nancing for Yong Wu highway: Excellent Master Thesis. Xi-an university of technology. (In Chinese)

Kumaraswamy, M.; Zhang, X. 2001. Governmental role in BOT-led infrastructure development, International Journal of Project Management 19(4): 195-205. http://dx.doi.org/10.1016/S0263-7863(99)00069-1

Ke, Y.; Wang, S.; Chan, A. 2008. Revelation of the Channel Tunnel's failure to risk allocation in Public-Private Partnership projects, China Civil Engineering Journal 41(12): 97-102. (In Chinese)

Khang, D. B.; Moe, T. L. 2008. Success criteria and factors for international development projects: a life-cycle-based framework, Project Management Journal 39(1): 72-84. http://dx.doi.org/10.1002/pmj.20034

Laishram, B.; Satyanarayana, N. 2009. Criteria influencing debt financing of Indian PPP road projects: a case study, Journal of Financial Management of Property and Construction 14(1): 34-60. http://dx.doi. org/10.1108/13664380910942635

Li, H. 2006. Financial appraisal and analysis of investment and financing mode of Nanjing crossing-river bridge project: Excellent Master Thesis. Southeast University. (In Chinese)

Liu, S. 2004a. BOT mode application practice and strategy study in China: Excellent Master Thesis. Sichuan University. (In Chinese)

Liu, Z. 2004b. Study on the utilization of BOT financing to facilitate the infrastructure construction of West China: Excellent Master Thesis. SiChuan University. (In Chinese)

Lu, H. 2006. Studies on the application of domesticinvestment BOT mode in sewage-disposal establishment in China: Excellent Master Thesis. Tianjin University. (In Chinese)

Lam, P. 1999. A sectoral review of risks associated with major infrastructure projects, International Journal of Project Management 17(2): 77-87. http://dx.doi. org/10.1016/S0263-7863(98)00017-9

Ma, Y. 2004. The application of BOT in infrastructure development of Yunnan provenience: Excellent Master Thesis. Kunming University of science and technology. (In Chinese).

Sobhiyah, M.; Bemanian M.; Kashtiban, Y. 2009. Increasing VFM in PPP power station projects - case study: Rudeshur gas turbine power station, International Journal of Project Management 27(5): 512521. http://dx.doi.org/10.1016/j.ijproman.2008.07.002

Song, J. B.; Song, D. R.; Zhang, X. Q.; Sun, Y. 2013. Risk identification for PPP waste-to-energy incineration projects in China, Energy Policy 61: 953-962. http:// dx.doi.org/10.1016/j.enpol.2013.06.041
Smith, N.; Zhang, H.; Zhu, Y. 2004. The Huaibei power plant and its implications for the Chinese BOT market, International Journal of Project Management 22(5): 407-413. http://dx.doi.org/10.1016/j.ijproman.2003.09.001

Tam, C. 1999. Build-operate-transfer model for infrastructure developments in Asian: reasons for successes and failures, International Journal of Project Management 17(6): 377-382. http://dx.doi. org/10.1016/S0263-7863(98)00061-1

Wang, S.; Tiong, L. 2000. Case study of government initiatives for PRC's BOT power plant project, International Journal of Project Management 18(1): 69-78. http://dx.doi.org/10.1016/S0263-7863(98)00072-6

Wang, X. 2001. The study of legal problem for risk allocation and government guarantees of BOT projects: Excellent Master Thesis. China University of Political Science and Legal. (In Chinese)

Wibowo, A. 2004. Valuing guarantees in a BOT infrastructure project, Engineering, Construction and Architectural Management 11(6): 395-403. http://dx.doi. org/10.1108/09699980410571543

Wibowo, A.; Permana, A.; Kochendörfer, B.; Kiong, R.; Jacob, D.; Neunzehn, D. 2012. Modeling contingent liabilities arising from government guarantees in Indonesian BOT/PPP toll roads, Journal of Construction Engineering and Management 138(12): 14031410. http://dx.doi.org/10.1061/(ASCE)CO.19437862.0000555

$\mathrm{Xu}$, H. Z. 2004. The assessment and management of risks associated with BOT project in China: Excellent Master Thesis. Jilin University. (In Chinese)

Xu, Y. L. 2010. Risk evaluation and allocation for PPP projects in China: $\mathrm{PhD}$ Thesis. Southeast University. (In Chinese)

Yeo, K. T. 2003. Managerial issues and lessons for the proposed Taiwan Strait Tunnel Project, Marine Georesources \& Geotechnology 21(3): 249-260. http:// dx.doi.org/10.1080/713773399

Yin, R. 2009. Case study research: design and methods. 4th ed. Beverly Hills, CA: Sage Publishing.

Zi, Y. T. 2005. An analysis of credit structure from the perspective of law: Excellent Master Thesis. China University of Political Science and Law. (In Chinese)

Zhou, X. L. 2004. Project financing model and credit guarantee structure study: Excellent Master Thesis. Wuhan University. (In Chinese)

Zhao, Y. P. 2002. Project financing of transportation infrastructure: Excellent Master Thesis. Xiamen University. (In Chinese)

Zhang, X. Q.; Kumaraswamy, M. M. 2001. Hong Kong experience in managing BOT projects, Journal of Construction Engineering and Management 127(2): 154-162. http://dx.doi.org/10.1061/(ASCE)07339364(2001)127:2(154)

Zhang, X. 2005. Paving the way for public-private partnerships in infrastructure development, Journal of Construction Engineering and Management 131(1): 71-80. http://dx.doi.org/10.1061/(ASCE)07339364(2005)131:1(71) 